\title{
La enfermería: ciencia constituida
}

Nursing: constituted science

https://doi.org/10.37135/ee.04.11.02

\section{Autoras:}

Nubia Blanco Balbeito' - https://orcid.org/0000-0002-0359-9157

Yovana Betancourt Roque ${ }^{1}$ - https://orcid.org/0000-0001-6533-5627

${ }^{1}$ Filial de Ciencias Médicas "Lidia Doce" de la Universidad de Ciencias Médicas de Villa Clara. Sagua la Grande - Cuba.

Autor de correspondencia: Nubia Blanco Balbeito. Email: gnubiabb@infomed.sld.cu. Dirección postal: Filial de Ciencias Médicas "Lidia Doce", Ave. 9 de Abril Km. 1 1/2, Sagua la Grande, Vílla Clara, Cuba.

\section{RESUMEN}

La autora realiza un proceso reflexivo acerca de la enfermería como ciencia constituida con sus propias teorías y modelos. Lo que argumenta con elementos históricos, epistemológicos y metodológicos.

Palabras clave: enfermería, ciencia, epistemología.

\section{ABSTRACT}

The author carries out a reflective process about nursing as a constituted science with its own theories and models, which is argued considering historical, epistemological, and methodological elements.

Keywords: Nursing, Science, Epistemology.

La enfermería es reconocida como la ciencia encargada del cuidado en salud humana, su evolución histórica transita desde una etapa meramente doméstica sin fundamentación teórica hasta constituirse en una profesión con sólidas bases epistemológicas. ${ }^{(1)}$

Esos sistemas de conocimientos también evolucionaron desde tradiciones relacionadas con el cuidado en entornos familiares, pasando por la recopilación de experiencias empíricas derivadas de la observación, hasta llegar a consolidar sus propias teorías y modelos establecidos mediante la aplicación del método científico. ${ }^{(2)}$ 
Los individuos constituyen en las principales unidades de observación durante los procesos investigativos relacionados con la enfermería. Así, a finales del siglo XX se produce un auge de estudios desde paradigmas cualitativos utilizando la fenomenología como base metodológica. Esto permite conocer las características del fenómeno a partir de las vivencias de las personas que forman parte de su manifestación..$^{(3)}$

Entre las técnicas para la recolección de los datos, la entrevista sobresale por su factibilidad en las investigaciones cualitativas con metodología fenomenológica. Desde la perspectiva particular, los individuos aportan su experiencia e interpretación del fenómeno estudiado. Luego el profesional de enfermería investigador realiza un análisis del corpus discursivo, aportando un valor agregado de humanización a los resultados, los que luego se reflejarán en los servicios del cuidado de salud humana. ${ }^{(4)}$

La tendencia actual indica la visión de un proceso enfermero con la capacidad de brindar un cuidado holístico, universal e intercultural al paciente, la familia y la comunidad. Los profesionales de la enfermería se han merecido un lugar destacado dentro de los equipos humanos de atención de salud.

Conflicto de intereses: la autora declara que no existe.

\section{Declaración de contribución:}

La autora realizó el proceso de búsqueda y selección de la información y elaboró el manuscrito a partir de un proceso reflexivo.

\section{REFERENCIAS BIBLIOGRÁFICAS}

1. Siles-González J. La construcción social de la Historia de la Enfermería. Index Enferm [Internet]. 2004 [citado 2020 Ago 12]; 13(47): 07-10. Disponible en: http://scielo.isciii. es/scielo.php?script=sci_arttext\&pid=S1132-12962004000300001\&lng=es.

2. Escobar-Castellanos B, Sanhueza-Alvarado O. Patrones de conocimiento de Carper y expresión en el cuidado de enfermería. Enfermería (Montevideo) [Internet]. 2018 [citado 2020 Ago 23]; 7(1): 27-42. Disponible en: http://www.scielo.edu.uy/scielo.php?script= sci_arttext\&pid=S2393-66062018000100027\&lng=es. http://dx.doi.org/10.22235/ech.v7i1. 1540 .

3. Expósito-Concepción MY, Villarreal-Cantillo E, Palmet-Jiménez MM, Borja-González JB, Segura-Barrios IM, Sánchez-Arzuza FE. La fenomenología, un método para el estudio del cuidado humanizado. Rev Cubana Enferm [Internet]. 2019 [citado 2020 Aago 21]; 
35(1): [aprox. 0 p.]. Disponible en: http://www.revenfermeria.sld.cu/index.php/enf/article/ view/2333.

4. Troncoso-Pantoja C, Amaya-Placencia A. Entrevista: guía práctica para la recolección de datos cualitativos en investigación de salud. Rev Fac Med [Internet]. 2017 [citado 2020 Ago 19];65: 329-332. Disponible en: https://revistas.unal.edu.co/index.php/revfacmed/ article/view/60235/62861 http://dx.doi.org/10.15446/revfacmed.v65n2.60235.

Recibido: 12 de febrero de 2021

Aprobado: 26 de abril de 2021 